\title{
Ações interdisciplinares do enfermeiro a crianças e adolescentes vítimas de violência sexual: revisão integrativa
}

\author{
Interdisciplinary actions of nurses to children and adolescents victims of sexual violence: an \\ integrative review
}

Acciones interdisciplinarias de enfermeras a niños y adolescentes víctimas de violencia sexual: una revisión integradora

Recebido: 18/10/2021 | Revisado: 26/10/2021 | Aceito: 27/10/2021 | Publicado: 30/10/2021

Welmer Danilo Rodrigues Rocha
ORCID: https://orcid.org/0000-0003-4495-2436
Instituto Federal de Educação, Ciência e Tecnologia do Tocantins, Brasil
E-mail: welmerrocha@gmail.com
Kamilla Germana da Silva
ORCID: https://orcid.org/0000-0002-8464-2013
Faculdade de Colinas do Tocantins, Brasil
E-mail: kamillager.s@gmail.com
Kyaren Sena da Silva Sousa
ORCID: https://orcid.org/0000-0001-7936-3311
Faculdade de Colinas do Tocantins, Brasil
E-mail: kyarensena7@gmail.com

\begin{abstract}
Resumo
Objetivo: O objetivo central desde estudo é identificar as evidências científicas nacionais e internacionais acerca das ações interdisciplinares do enfermeiro a crianças e adolescentes vítimas de violência sexual. Método: Revisão integrativa da literatura, realizada em quatro bases de dados, com os critérios de inclusão: tipo de estudo, idioma e recorte temporal. A amostra final foi composta por 10 estudos. Resultados: Da síntese das evidências, emergiram quatro categorias: rede de atendimento, profissional de saúde na rede de atendimento, protocolos e serviços; organizadas em potencialidades, demandas e fragilidades. Constituindo elementos necessários para a eficácia das ações interdisciplinares do enfermeiro a crianças e adolescentes vítimas de violência sexual. Conclusão: Contudo, a construção desse estudo aporte a importância da aptidão e competência da equipe de enfermagem, principalmente o enfermeiro acerca de serviço prestado as vítimas, com ênfase nas políticas públicas.
\end{abstract}

Palavras-chave: Violência sexual; Criança e adolescente; Enfermagem.

\begin{abstract}
Objective: The main objective of this study is to identify national and international scientific evidence about the interdisciplinary actions of nurses to children and adolescents victims of sexual violence. Method: Integrative literature review, carried out in four databases, with the inclusion criteria: type of study, language and time frame. The final sample consisted of 10 studies. Results: From the synthesis of evidence, four categories emerged: service network, health professional in the service network, protocols and services; organized in strengths, demands and weaknesses. Constituting necessary elements for the effectiveness of interdisciplinary actions by nurses to children and adolescents victims of sexual violence. Conclusion: However, the construction of this study contributes to the importance of the aptitude and competence of the nursing team, especially nurses regarding the service provided to victims, with an emphasis on public policies.
\end{abstract}

Keywords: Sexual violence; Child and teenager; Nursing.

\section{Resumen}

Objetivo: El objetivo principal de este estudio es identificar evidencia científica nacional e internacional sobre las acciones interdisciplinarias de las enfermeras a los niños y adolescentes víctimas de violencia sexual. Método: revisión integrativa de la literatura, realizada en cuatro bases de datos, con los criterios de inclusión: tipo de estudio, idioma y marco temporal. La muestra final consistió en 10 estudios. Resultados: De la síntesis de evidencia surgieron cuatro categorías: red de servicios, profesional de la salud en la red de servicios, protocolos y servicios; organizados en fortalezas, demandas y debilidades. Constituir elementos necesarios para la efectividad de las acciones interdisciplinarias de las enfermeras a los niños, niñas y adolescentes víctimas de violencia sexual. Conclusión: Sin embargo, la construcción de este estudio contribuye a la importancia de la aptitud y competencia del equipo de 
enfermería, especialmente las enfermeras, en cuanto al servicio brindado a las víctimas, con énfasis en las políticas públicas.

Palabras clave: Violencia sexual; Niño y adolescente; Enfermería.

\section{Introdução}

A violência sexual é um tema de grande complexidade, de difícil análise, e também, apresenta dificuldade para evidenciar os acontecimentos que são vividos na sociedade, pelo fato de ser um acontecimento indesejado, devido acarretar consequências negativas que intervém na vida do ser humano mediante ao desenvolvimento psicossocial das crianças e adolescentes, nas relações interpessoais, tornando-se transtornos que irão limitar ou alterar sua conduta e seu comportamento (Nunes, 2021). Dessa forma é necessário que as medidas contra violência sexual sejam conduzidas de modo coordenado entre os serviços das redes de proteção e de atendimento por profissionais capacitados (Hohendorff \& Patias, 2017).

Logo, a violência sexual classifica-se em dois momentos como o abuso que é o uso da sexualidade e a exploração proporcionada para fins lucrativos, configurando-se $11 \%$ das denúncias que se referem a este grupo específico (crianças e adolescentes), correspondendo a 17 mil de ocorrência, sucedendo a um aumento de 14 \% em relação a 2018. (Brasil, 2020).

Neste sentido e buscando uma reflexão mais ampla da problemática o foco deste estudo é descrever a violência sexual, e suas consequências mediante as fases da existência humana, pois segundo Pintor (2017, p.16) o impacto psicológico/emocional depende do caso concreto, e poderá ser mediada pelo suporte familiar, e pelo contexto ambiental, de modo a atenuar os efeitos, norteando para uma produção de conhecimento de alerta e desafios do corpo e a sexualidade.

Dessa forma, levando ao enfermeiro a ter sempre um conhecimento de forma variada, apresentando o papel de favorecer a motivação individual do sujeito, que sofre psiquicamente, para que ele possa se desenvolver como pessoa, respeitando-se a si e aos outros, através do relacionamento interpessoal (Moreira, 2020).

De acordo com Soares (2021), o termo Adverse Childhood Experiences (ACEs) conhecido no Brasil como Experiências Adversas na Infância está associado às várias consequência traumáticas que ocorrem na infância. E tem como objetivo compreender aos eventos traumáticos é as possíveis causas de estresse durante o período infanto-juvenil, abrangendo situações como o abuso (emocional, físico ou sexual) (Pedrosa, 2018).

A Resolução COFEN (564/2017) aprova o Código de Ética dos profissionais de enfermagem que autoriza o dever de comunicação externa para os órgãos de responsabilização criminal, independente de autorização dos responsáveis, relacionados a casos de violência contra: crianças e adolescentes. Consequentemente, podendo resultar no acolhimento e proteção da vítima e familiares. Esse dever se fundamenta no conhecimento próprio da profissão, nas ciências humanas, sociais e aplicadas.

Explanando sobre tipo de violência sexual que está disposta na sociedade atual, caracterizando uma análise e a possibilidade de ações de interdisciplinaridades abrindo espaços de diálogos, debates e estratégias em que o enfermeiro é capaz de intervir na promoção e prevenção da saúde de crianças e adolescentes.

Portanto, o enfermeiro tem o papel de compreender o contexto de violência sexual, adquirindo através de conhecimento especifico e qualificado para o manejo clínico e psicológico (Teixeira, 2019). Assim a pesquisa questiona-se com base nas evidências científicas, quais as ações de interdisciplinares em que o enfermeiro é capaz de intervir a crianças e adolescentes vítimas de violência sexual? O objetivo central desde estudo é identificar as evidências científicas nacionais e internacionais acerca das ações interdisciplinares do enfermeiro a crianças e adolescentes vítimas de violência sexual. Acredita-se que a relevância deste estudo é relatar as ações conjuntas e intersetoriais do enfermeiro evidenciadas em programas que atendem a situações de violência sexual a crianças e adolescentes. 


\section{Metodologia}

Trata-se de uma revisão integrativa da literatura, com intuito de identificar a produção científica a respeito das ações interdisciplinares praticadas pela enfermagem que é capaz de intervir na saúde mental de crianças e adolescentes vítimas de violência sexual, assim considerando a relevância social para a enfermagem e sociedade. (Quaglio; Bueno \& Almeida, 2017).

Para tanto, foram selecionados os descritores no DeCS/Mesh (Descritores em Ciências da Saúde/Medical Subject Headings): Violência sexual / sexual violence, criança e adolescente / child and teenager e enfermagem / nursing, em inglês, espanhol e português. As bases de dados selecionadas foram PUBMED/MEDILINE, Biblioteca Virtual em Saúde (BVS) e na Literatura Latino-Americana e do Caribe em Ciências da Saúde (LILACS).

A busca procedeu-se no mês de agosto de 2021. Vale ressaltar que foram aplicadas estratégias de busca distintas em cada base de dados e na biblioteca digital, sendo identificados 781 artigos relacionados com a temática desta pesquisa. Optouse por delimitar a análise dos artigos incluindo apenas aqueles publicados nos últimos 6 anos de 2016 a 2021. Os critérios de inclusão decorreram: artigos na íntegra, acesso gratuito, disponível on-line, idiomas em inglês, espanhol e português.

Como critério de exclusão: artigo não disponível na integra, não abordavam a temática, publicados em outros idiomas exceto inglês e português, teses, resumos, dissertações, estudos de revisão, estudos duplicados, resenhas, reportagens, notícias e editoriais.

Ao fim, as categorias foram organizadas conforme o intuito deste estudo, totalizando a leitura dos 18 artigos na íntegra, destes, 8 foram excluídos por não responder à questão norteadora da revisão. A amostra final foi composta por 10 estudos, que foram organizados em uma tabela no Microsoft Word® para a síntese, a partir dos principais resultados e agrupando-os por categorias. O esquema de busca e seleção dos estudos é apresentado na Figura 1.

Além disso, buscou-se dados nos manuais disponíveis na internet do Estatuto da Criança e do Adolescente (ECA), com conteúdo na importância da atuação da enfermagem diante os cuidados a crianças e adolescentes vítima de violência sexual, amparado pela Lei no 8.069, de 13 de julho de 1990, e realizar o acolhimento e proteção da vítima e familiares, em caso de violência contra: crianças e adolescentes na Resolução COFEN (564/2017). 
Figura 1 - Fluxograma de descrição dos artigos, encontrados, excluídos e selecionados de acordo com a cada base de dados.

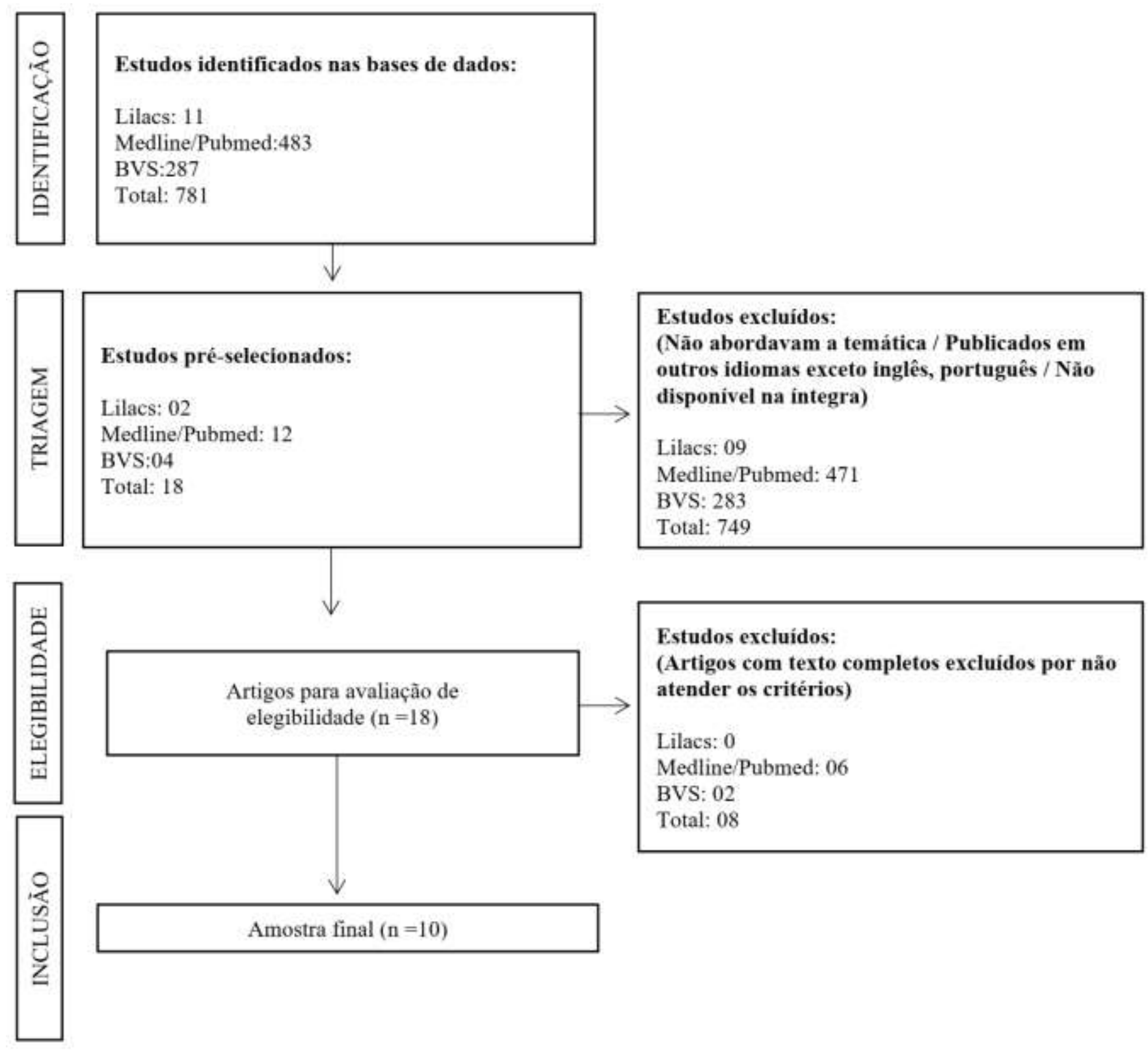

Fonte: Autores.

\section{Resultados}

Quanto à caracterização dos 10 estudos selecionados, o número de publicações por ano foi: 2016: um; 2017: um; 2018: um; 2019: três; 2020: três; 2021: um (até a data da busca). Em relação ao país em que foram desenvolvidos os estudos: Brasil (03), Estados Unidos da América (01), Canadá (01); África do Sul (02); Reino Unido (02) e Austrália (01). O idioma predominante nas publicações foi o inglês (07), seguido do português (03). Referente à abordagem metodológica: estudos qualitativos (02), estudos de revisão sistemática (04), estudo ecológico (01), estudos de revisão abrangente (01), estudos de revisão sistemática e metanálise (01) e estudos métodos mistos (01). O Quadro 1 apresenta o ano de publicação, país, título e objetivo dos estudos selecionados. 
Quadro 1. Caracterização dos estudos acerca das ações interdisciplinar do enfermeiro na saúde mental das crianças e adolescente frente à violência sexual, segundo: ano de publicação, país, título e objetivo.

\begin{tabular}{|c|c|c|c|}
\hline Ano & País & Título & Objetivo \\
\hline 2020 & Reino Unido & $\begin{array}{l}\text { Association of Childhood Maltreatment } \\
\text { With Suicide Behaviors Among Young } \\
\text { People A Systematic Review and Meta- } \\
\text { analysis. }\end{array}$ & $\begin{array}{l}\text { Para quantificar a associação entre os tipos } \\
\text { de tratamento infantil, incluindo abuso } \\
\text { sexual, físico e emocional e/ou } \\
\text { comportamentos suicidas e suicidas em } \\
\text { crianças e adultos jovens. }\end{array}$ \\
\hline 2017 & Canadá & $\begin{array}{l}\text { Rethinking research on sexual exploitation } \\
\text { of boys: Methodological challenges and } \\
\text { recommendations tooptimize future } \\
\text { knowledge generation. }\end{array}$ & $\begin{array}{l}\text { Identificar e descrever os principais desafios } \\
\text { metodológicos da literatura, a fim de } \\
\text { informar pesquisadores e clínicos sobre os } \\
\text { limites das pesquisas existentes sobre a } \\
\text { exploração sexual de meninos. }\end{array}$ \\
\hline 2020 & Austrália & $\begin{array}{l}\text { Demographic and Psychosocial Factors } \\
\text { Associated With Child Sexual Exploitation } \\
\text { A Systematic Review and Meta-analysis. }\end{array}$ & $\begin{array}{l}\text { Fornecer uma síntese meta-analítica de } \\
\text { estudos que examinam os fatores associados } \\
\text { à ESC e quantificar sua importância relativa. }\end{array}$ \\
\hline 2020 & Brasil & $\begin{array}{l}\text { "Não tenho, não tive": vivências de } \\
\text { famílias envolvidas na violência contra } \\
\text { crianças e adolescentes. }\end{array}$ & $\begin{array}{l}\text { Caracterizar e analisar as vivências de } \\
\text { famílias envolvidas na violência doméstica } \\
\text { contra crianças e adolescentes, com base no } \\
\text { Paradigma da Complexidade. }\end{array}$ \\
\hline 2018 & África do Sul & $\begin{array}{l}\text { Sexual assault survivors' perspectives on } \\
\text { clinical follow-up in the Eden District, } \\
\text { South Africa: A qualitative study. }\end{array}$ & $\begin{array}{l}\text { Este estudo explorou as experiências } \\
\text { pessoais de sobreviventes de agressão sexual } \\
\text { para melhor compreender os facilitadores e } \\
\text { as barreiras para o comparecimento às } \\
\text { consultas de acompanhamento. }\end{array}$ \\
\hline 2019 & Brasil & $\begin{array}{l}\text { Violência sexual contra crianças e } \\
\text { adolescentes: uma análise da prevalência e } \\
\text { fatores associados. }\end{array}$ & $\begin{array}{l}\text { Analisar a prevalência e os fatores } \\
\text { associados à violência sexual contra crianças } \\
\text { e adolescentes da cidade de Petrolina / } \\
\text { Pernambuco. }\end{array}$ \\
\hline 2021 & Brasil & $\begin{array}{l}\text { Desafios da atuação do enfermeiro frente à } \\
\text { violência sexual infanto-juvenil. }\end{array}$ & $\begin{array}{l}\text { Identificar a percepção de enfermeiros } \\
\text { quanto aos desafios enfrentados durante sua } \\
\text { atuação frente à violência sexual infanto- } \\
\text { juvenil. }\end{array}$ \\
\hline 2019 & Reino Unido & $\begin{array}{l}\text { Long-term outcomes of childhood sexual } \\
\text { abuse: an umbrella review. }\end{array}$ & $\begin{array}{l}\text { Revisar sistematicamente as meta-análises } \\
\text { existentes em uma ampla gama de resultados } \\
\text { psiquiátricos, psicossociais e físicos de longo } \\
\text { prazo do abuso sexual na infância e avaliar a } \\
\text { qualidade da literatura. }\end{array}$ \\
\hline 2016 & USA & $\begin{array}{l}\text { Revisiting Child Sexual Abuse and } \\
\text { Survivor Issues. }\end{array}$ & $\begin{array}{l}\text { Informação sobre o que os enfermeiros } \\
\text { podem fazer caso suspeitam que uma criança } \\
\text { foi ou está sendo abusada. }\end{array}$ \\
\hline 2019 & África do Sul & $\begin{array}{l}\text { Adverse childhood experiences (ACEs) are } \\
\text { associated with forced and very early } \\
\text { sexual initiation among Black women } \\
\text { accessing publicly funded STD clinics in } \\
\text { Baltimore, MD. }\end{array}$ & $\begin{array}{l}\text { Examinar a associação entre experiências } \\
\text { adversas na infância (ACEs) e iniciação } \\
\text { sexual precoce. }\end{array}$ \\
\hline
\end{tabular}

Fonte: Autores.

A partir da leitura e da comparação entre os resultados dos estudos, emergiram quatro categorias, organizadas em: competências, demandas e debilidades no atendimento a crianças e adolescentes em situação de violência sexual. Essas categorias são apresentadas no Quadro 2. 
Quadro 2. Competências, demandas e Debilidades identificadas nos estudos selecionados.

\begin{tabular}{|c|c|c|c|}
\hline \multirow[b]{2}{*}{ 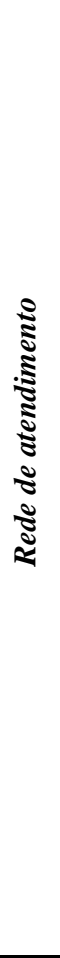 } & Competências & Demandas & Debilidades \\
\hline & $\begin{array}{l}\text { Promoção da saúde, prevenção de } \\
\text { violências e proteção dos direitos de } \\
\text { crianças e adolescentes (Miranda et al., } \\
\text { 2019). } \\
\text { Implementação de serviço psicossocial } \\
\text { (Holton; Joyner \& Mash, 2018). } \\
\text { Associação entre experiências adversas na } \\
\text { infância (ACEs) (Tsuyuki et al., 2019). }\end{array}$ & $\begin{array}{l}\text { Articulação dos serviços de saúde com demais } \\
\text { serviços, de assistência social, educação e com } \\
\text { os sistemas de justiça, segurança pública, } \\
\text { Ministério Público, Defensoria Pública, Varas } \\
\text { da Infância e Juventude, Conselho Tutelar, } \\
\text { conselhos de direitos, e a sociedade civil } \\
\text { organizada, para implementar e fortalecer a } \\
\text { rede de cuidado e proteção social. (Miranda et } \\
\text { al., 2019). } \\
\text { Intersetorialidade, quando enfermeiros (as) } \\
\text { compartilham suas preocupações e constroem } \\
\text { respostas junto com profissionais da assistência } \\
\text { social, ajudam a prevenir a exposição de } \\
\text { crianças e adolescentes a novos riscos (Carlos } \\
\text { et al., 2020). } \\
\text { Prevenção primária do abuso sexual infantil } \\
\text { tem se concentrado principalmente em } \\
\text { intervenções na escola e nas intervenções dos } \\
\text { pais em casa (Sabella D., 2016). } \\
\text { As perguntas devem ser realizadas de acordo } \\
\text { com a idade da criança e do nível cognitivo e de } \\
\text { maturidade (Sabella D., 2016). }\end{array}$ & $\begin{array}{l}\text { Tem as Educações em Saúde. Mas } \\
\text { específico para a violência sexual não } \\
\text { tem (Silva et al., 2021). } \\
\text { Revitimização (Carlos et al., 2020). } \\
\text { Fragilidade dos serviços públicos de } \\
\text { saúde em acolher e acompanhar } \\
\text { (Miranda et al., 2019). } \\
\text { Os profissionais informam não estarem } \\
\text { aptos para esse tipo de atendimento } \\
\text { (Silva et al., 2021). } \\
\text { Necessário maior apoio governamental } \\
\text { para que os profissionais da } \\
\text { enfermagem possam se sentir mais } \\
\text { seguros ao atuar nesses casos (Silva et } \\
\text { al., 2021). } \\
\text { Estudos de qualidade insuficiente para } \\
\text { orientar de forma significativa a } \\
\text { prevenção e intervenção (Laird et al., } \\
\text { 2020). }\end{array}$ \\
\hline \multirow[b]{2}{*}{ 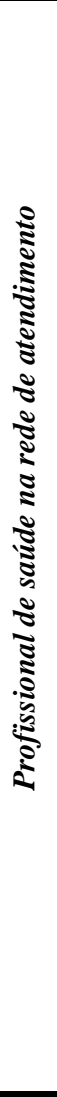 } & Competências & Demandas & Debilidades \\
\hline & $\begin{array}{l}\text { Enfermeiras interpretam que para intervir } \\
\text { nos casos de violência, além do } \\
\text { conhecimento atualizado, a atuação deve ser } \\
\text { interdisciplinar (Silva et al., 2021). } \\
\text { Intervenções apropriadas com melhores } \\
\text { respostas a crianças, adolescentes e suas } \\
\text { famílias (Carlos et al., 2020). } \\
\text { Cuidado individualizado (Holton; Joyner \& } \\
\text { Mash, 2018). } \\
\text { Triagem é fundamental para prevenir uma } \\
\text { maior exploração sexual de crianças e } \\
\text { adolescentes vulneráveis (Laird et al., } \\
\text { 2020). } \\
\text { Faz a criança identificar suas partes do } \\
\text { corpo, determinam quais palavras à criança } \\
\text { usa para as partes do corpo, discute com a } \\
\text { criança o conceito de partes íntimas e } \\
\text { pergunta se alguém toca na criança lá, se a } \\
\text { criança responder (sim) á pergunta anterior, } \\
\text { ela investiga quem toca a criança e onde e } \\
\text { como e com adolescentes, ela discute a } \\
\text { atividade sexual consensual e nâ } \\
\text { consensual e as práticas sexuais seguras } \\
\text { (Sabella D., 2016). }\end{array}$ & $\begin{array}{l}\text { São poucos os programas ministeriais } \\
\text { conhecidos pelos profissionais e aderidos pelas } \\
\text { gestões municipais, estaduais e federais a fim } \\
\text { de propiciar meios para o combate da violência } \\
\text { sexual (Silva et al., 2021). }\end{array}$ & $\begin{array}{l}\text { Dificuldades dos profissionais no } \\
\text { manejo da violência contra a criança e } \\
\text { ao adolescente (Silva et al., 2021). } \\
\text { Ineficiência de incentivos a cursos e } \\
\text { profissionais especializados } \\
\text { ocasionando a insegurança no } \\
\text { atendimento (Silva et al., 2021). } \\
\text { Escassez de pesquisas na área de CSE } \\
\text { (Laird et al., 2020). } \\
\text { Desafios para o atendimento contínuo } \\
\text { nos serviços atuais (Holton; Joyner \& } \\
\text { Mash, 2018). } \\
\text { Vulnerabilidade social (Carlos et al., } \\
\text { 2020). } \\
\text { São necessários estudos e ações que } \\
\text { coordenem e integrem respostas } \\
\text { voltadas para o reconhecimento dessas } \\
\text { conexões e dimensões (Carlos et al., } \\
\text { 2020). }\end{array}$ \\
\hline \multirow[b]{2}{*}{ 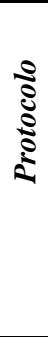 } & Competências & Demandas & Debilidades \\
\hline & $\begin{array}{l}\text { Protocolo Opcional sobre o Venda de } \\
\text { crianças, prostituição infantil e pornografia } \\
\text { infantil. (Michell et al, 2017). } \\
\text { As informações podem ser obtidas no } \\
\text { conselho de enfermagem do estado, e as } \\
\text { diretrizes do estado estão disponíveis no site } \\
\text { da Rede Nacional de Estupro, Abuso e }\end{array}$ & $\begin{array}{l}\text { É preciso construir um olhar ampliado sobre a } \\
\text { violência e sobre os envolvidos nela (Carlos et } \\
\text { al., 2020). } \\
\text { Falta de articulação dos serviços (Silva et al., } \\
2021 \text { ). }\end{array}$ & $\begin{array}{l}\text { Há pouco detalhamento teórico de } \\
\text { programas e manuais que reportam } \\
\text { sobre a violência sexual, fluxograma de } \\
\text { atendimento e prevenção do evento } \\
\text { (Silva et al., 2021). } \\
\text { Não sabe com intervir nos casos de } \\
\text { violência sexual de modo a informar às }\end{array}$ \\
\hline
\end{tabular}




\begin{tabular}{|c|c|c|c|}
\hline & $\begin{array}{l}\text { Incesto (Sabella D., 2016). } \\
\text { Compreensão do processo de ocorrência } \\
\text { deste evento, bem como a formação de uma } \\
\text { equipe profissional que seja capaz de atuar } \\
\text { com competência e compromisso para que } \\
\text { haja o enfrentamento destes casos (Silva et } \\
\text { al., 2021). }\end{array}$ & & $\begin{array}{l}\text { autoridades competentes sobre o } \\
\text { ocorrido (Silva et al., 2021). } \\
\text { Inexistência de protocolos (Silva et al., } \\
\text { 2021). } \\
\text { Não estar habilitada para lidar com } \\
\text { situações de violência, o que pode levar } \\
\text { à invisibilidade de casos (Silva et al., } \\
\text { 2021). }\end{array}$ \\
\hline & Competências & Demandas & Debilidades \\
\hline 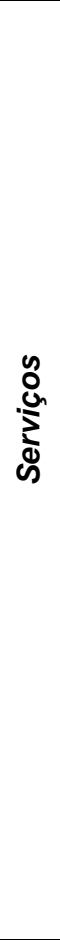 & $\begin{array}{l}\text { Propedêutica deve incluir uma equipe } \\
\text { multiprofissional no contexto familiar (Silva } \\
\text { et al., 2021). } \\
\text { Prestação de uma assistência equânime e de } \\
\text { qualidade, sendo esta diferenciada e } \\
\text { individualizada (Silva et al., 2021). } \\
\text { Divulgação de violência no SINAN, como } \\
\text { ferramenta eletrônica para combater a } \\
\text { violência (Miranda et al., 2019). } \\
\text { Identificar sinais e sintomas de violências, } \\
\text { efetuando o atendimento, acolhimento } \\
\text { notificação dos casos e encaminhamento das } \\
\text { vítimas na rede de cuidados (Miranda et al., } \\
\text { 2019). } \\
\text { Contribuir na (re)significação das histórias } \\
\text { violentas tecidas por famílias (Carlos et al., } \\
\text { 2020). } \\
\text { Enfermagem possui um papel-chave na } \\
\text { identificação, manejo e tomada de decisões } \\
\text { junto com serviços de proteção (Carlos et } \\
\text { al., 2020). }\end{array}$ & $\begin{array}{l}\text { Desafios a políticas públicas (Carlos et al., } \\
\text { 2020). } \\
\text { Não ocorrem ações de prevenção (Carlos et al., } \\
\text { 2020). } \\
\text { Organização das equipes, da rede de justiça e } \\
\text { das Organizações Não-Governamentais (ONG) } \\
\text { quanto os cuidados com os adolescentes (Silva } \\
\text { et al., 2021). }\end{array}$ & $\begin{array}{l}\text { Não estarem aptos para esse tipo de } \\
\text { atendimento (Silva et al., 2021). } \\
\text { Insegurança e dificuldades de agir } \\
\text { diante das situações de violência sexual } \\
\text { infanto-juvenil (Silva et al., 2021). } \\
\text { Precarização das condições de um } \\
\text { atendimento efetivo à vítima oferecida } \\
\text { pelo sistema de gestão vigente (Silva et } \\
\text { al., 2021). } \\
\text { Barreiras entre o cliente e o serviço } \\
\text { (Holton; Joyner; Mash, 2018). } \\
\text { Falta de privacidade (Holton; Joyner \& } \\
\text { Mash, 2018). } \\
\text { Incapacidade da polícia, justiça e de } \\
\text { segurança (Holton; Joyner \& Mash, } \\
\text { 2018). }\end{array}$ \\
\hline
\end{tabular}

Fonte: Autores.

\section{Discussão}

Nos estudos selecionados, algumas questões se destacam as ações do enfermeiro na saúde mental das crianças e adolescente frente à violência sexual. A rede de atendimento é uma dessas questões, quando implementa no serviço de atendimento psicossocial com serviços de promoção da saúde, prevenção de violências e proteção dos direitos de crianças e adolescentes torna-se uma competência no atendimento (Miranda et al., 2019). Entretanto, estudos indicam debilidades na educação em saúde da rede, fragilidades nos serviços públicos de saúde em acolher e acompanhar e no apoio governamental para segurança profissional aos atendimentos (Sabella 2016). Essas lacunas, como na educação em saúde ou os profissionais que não se sentem aptos para seus atendimentos, implica em consequências como a revitimização (Carlos et al., 2020).

Outro ponto importante é a (ACEs), a prevalência criticamente alta da iniciação sexual precoce e forçada (Tsuyuki et al., 2019). Órgãos como Ministério Público, Defensoria Pública, Varas da Infância e Juventude, Conselho Tutelar, Conselhos de Direitos e Sociedade Civil Organizada trabalham em implementar e fortalecer a rede de cuidado e proteção social (Miranda et al., 2019). Mas ao mesmo tempo a articulação de apoio entre enfermeiro e os órgãos governamentais reflete na insuficiência de orientação resultando de forma significativa na prevenção e intervenção (Laird et al., 2020

O trabalho do profissional da saúde especialmente do enfermeiro demanda intersetorialidade, compartilhando suas preocupações e construindo respostas junto a outros profissionais, como exemplo a equipe de assistência social que ajudam a prevenir a exposição de crianças e adolescentes a novos riscos (Carlos et al., 2020). No entanto estudos revelam que uma 
grande parcela de profissionais informa não se sentirem aptos a prestar este tipo de atendimento. Percebendo assim uma necessidade de apoio aos profissionais de enfermagem em educação permanente para que assim possam se sentir mais seguros e qualificados ao atuar nesses casos (Silva et al., 2021).

Para o profissional de saúde na rede de atendimento, expondo-se como competência a importância de um plano de cuidados individualizado para cada paciente, no qual o enfermeiro identifica as ações necessárias para que o paciente alcance resultados esperados (Holton; Joyner \& Mash, 2018). Trazendo assim, intervenções apropriadas com melhores respostas a crianças, adolescentes e suas famílias (Carlos et al., 2020). No entanto são necessários estudos e ações que coordenem e integrem respostas voltadas para o reconhecimento da necessidade de interpretações e intervenções que levam em conta as múltiplas violências, visto que é preciso preparar os profissionais para perceber sobre a violência (Carlos et al., 2020).

Além disso, profissionais de saúde interpretam que para intervir nos casos de violência, além do conhecimento atualizado, a atuação deve ser interdisciplinar, como competência no atendimento (Silva et al., 2021). Entretanto, a vulnerabilidade social afeta grande parte das famílias e apontam desafios para o atendimento contínuo nos serviços atuais, causando assim dificuldades dos profissionais no manejo da violência contra a criança e ao adolescente (Holton, Joyner \& Mash, 2018).

Dessa forma, a triagem é fundamental para prevenir o aumento estatístico de exploração sexual de crianças e adolescentes vulneráveis (Laird et al., 2020). Além disso, a performance da enfermagem na interação com a criança e os pais, faz com que o diálogo seja compreensível trazendo assim para a criança uma linguagem corporal para conhecer a si mesma com a necessidade de enfatizar, a partir de observações, a necessidade de a criança conhecer as funções de seu corpo (Sabella D., 2016). Porém o baixo incentivo a cursos especializados ocasiona a insegurança no atendimento pelo fato de serem poucos os programas ministeriais conhecidos pelos profissionais e aderidos pelas gestões municipais, estaduais e federais a fim de propiciar meios para o combate da violência sexual (Silva et al., 2021).

Referente aos protocolos, verifica-se como competências, a compreensão do processo de ocorrência deste evento, bem como a formação de uma equipe profissional que seja capaz de atuar com capacidade e compromisso para que haja o enfrentamento destes casos (Silva et al., 2021). A propósito, é preciso construir um olhar ampliado sobre a violência e sobre os envolvidos nela, para que não haja falta de articulação dos serviços (Carlos et al., 2020). Dessa forma, há pouco detalhamento teórico de programas e manuais que reportam sobre a violência sexual, fluxograma de atendimento e prevenção do evento (Silva et al., 2021).

Logo, as informações podem ser obtidas no conselho de enfermagem do estado, e as diretrizes do estado estão disponíveis no site da Rede Nacional de Estupro, Abuso e Incesto (Sabella 2016). Dessa forma, o profissional de enfermagem estar habilitada para lidar com situações de violência, porém a maioria não se sente aptos diante de casos de abuso sexual levando à invisibilidade de casos (Silva et al., 2021).

Por fim, percebe-se através dos estudos que é quase que inexistente o número de capacitação ou preparo dos profissionais em notificar a violência no exercício da sua profissão, o que contribui para a não notificação. Dessa forma, com a ausência de protocolos, grande parte dos profissionais não sabem como intervir nos casos de violência sexual de modo a informar às autoridades, ou seja, notificar e encaminhar para o hospital de referência assim como, para o Conselho Tutelar e, em seguida, acionar o Centro de Referência Especializada em Assistência Social (CREAS) e realizar o Boletim de Ocorrência por meio de uma Unidade Policial, em cada fase de seu crescimento e desenvolvimento (Silva et al., 2021).

Outro fator importante são os serviços, a enfermagem possui um papel-chave na identificação, manejo e tomada de decisões junto com serviços de proteção, que contribui na (re)significação das histórias violentas tecidas por famílias (Carlos et al., 2020). Por outro lado, apresenta desafios no apoio relacionado a políticas públicas (Carlos et al., 2020). Consequentemente gerando a insegurança e dificuldades de agir diante das situações de violência sexual infanto-juvenil (Silva et al., 2021). 
Desse modo, a competência do enfermeiro é identificar sinais e sintomas de violências, efetuando o atendimento, acolhimento, notificação dos casos de violência no SINAN, como ferramenta eletrônica para combater a violência, e encaminhamento das vítimas na rede de cuidados (Miranda et al., 2019). Some-se a isto, a importância da organização das equipes, da rede de justiça e das Organizações Não-Governamentais (ONG) quanto os cuidados com os adolescentes (Silva et al., 2021). No entanto, os profissionais evidenciam barreiras entre o cliente e o serviço, como a falta de privacidade, incapacidade do poder de polícia, justiça e de segurança local de atendimento (Holton; Joyner \& Mash, 2018).

Por muitas vezes não ocorrem ações de prevenção e aperfeiçoamento dos profissionais (Carlos et al., 2020). As equipes declaram não estarem aptos para esse tipo de atendimento, assim como precarização das condições de um atendimento efetivo à vítima oferecida pelo sistema de gestão vigente sendo que é essencial a prestação de uma assistência equânime e de qualidade, diferenciada e individualizada disposto por uma equipe multiprofissional (Silva et al., 2021).

Algumas considerações acerca do atendimento à saúde mental das crianças e adolescente frente à violência sexual no contexto. Compreende-se nos estudos a necessidade de capacitação dos profissionais para assegurar uma assistência propícia e de qualidade com perspectiva na integralidade das ações, assim como a desarticulação entre os serviços. Em razão dos profissionais não serem habilitados para lidar com situações de violência sexual, o que pode levar à invisibilidade dos casos.

Acrescentam-se também, além dos aspectos já mencionados, as dificuldades de acesso e de acessibilidade das crianças e adolescentes ao atendimento se tornam maiores, por falta de não ocorrerem ações de prevenção, desinformação ao familiar/cuidado, entre outros fatores. Dessa forma, é preciso construir um olhar ampliado sobre a violência e sobre os envolvidos nela, com preparo na intervenção adequada nos casos de violência sexual de modo a informar às autoridades competentes sobre o ocorrido.

Diante das adversidades do estudo, destaca-se como limitações o baixo número de estudo relacionado à saúde mental das crianças e adolescentes frente à violência sexual, principalmente com atuação das ações interdisciplinares do enfermeiro.

\section{Conclusão}

A revisão expõe uma noção do contexto acerca das ações interdisciplinares do enfermeiro ao atendimento às crianças e adolescentes em situação de violência sexual. Nos estudos selecionados, identificamos competências, debilidades e demandas. Ainda que competências, como qualidade do atendimento clínico por meio de protocolos, treinamentos e acolhimento; existem debilidades, como despreparo profissional na abordagem, já que não se encontram aptos para esse tipo de atendimento, ausência de protocolos, estudos de qualidade insuficiente, serviços inadequados, dificuldades de acesso, que desafiam a concretização do atendimento integral.

Nos estudos selecionados, identificamos quais a ações interdisciplinares e estratégias utilizadas na assistência de enfermagem perante á crianças e adolescentes vítimas de abuso sexual. Dessa forma, segmentamos 10 artigos dentro da temática abordada. A partir disso, constatam-se lacunas referentes ausência de protocolos, estudos de qualidade insuficiente, serviços inadequados, dificuldades de acesso que desafiam a concretização do atendimento integro.

Contudo, a construção desse estudo aporte a importância da aptidão e competência da equipe de enfermagem, principalmente o enfermeiro acerca de serviço prestado as vítimas, com ênfase nas políticas públicas. Apontando em estratégias de orientação, prevenção e acompanhamento. Sugerindo aos novos estudos abordar as intervenções empregadas pela equipe multiprofissional de saúde em crianças e adolescentes vítimas de abuso sexual.

Sugere-se, a importância de realizar estudos específicos na área da violência sexual a crianças e adolescentes. A identificação da violência é o primeiro passo para se adotar estratégias que protejam pessoas vulneráveis, tendo em vista que, geralmente, há sinais de abusos e os profissionais de saúde devem estar atentos para que possam adotar medidas de proteção.

Assim a revisão demonstra a seriedade do enfermeiro em ações interdisciplinares em combate ao abuso sexual de 
crianças e adolescentes para uma assistência de prevenção, intervenções, proteção e defesa dos direitos de crianças e adolescentes, com base nas superações de medos, transtornos e traumas. Como também, faz-se necessário a importância de capacitações e treinamentos, para que os profissionais da enfermagem possam se sentir mais seguros ao atuar nesses casos.

\section{Referências}

Angelakis, I., Austin, J. L., \& Gooding, P. (2020). Association of Childhood Maltreatment With Suicide Behaviors Among Young People A Systematic Review and Meta-analysis. https://jamanetwork.com/journals/jamanetworkopen/fullarticle/2769030

Brasil. (2017). Conselho Federal de Enfermagem (BR). Resolução N ${ }^{\circ} 564$ Conselho Federal de Enfermagem, 06 de dezembro de 2017 (BR). http://www.cofen.gov.br/resolucao-cofen-no-5642017_59145.html

Brasil. (2020). Ministério da Saúde (BR). Ministério divulga dados de violência sexual contra crianças e adolescentes (BR). https://www.gov.br/mdh/ptbr/assuntos/noticias/2020-2/maio/ministerio-divulga-dados-de-violencia-sexual-contra-criancas-e-adolescentes

Brasil. (1990). Presidência da República Casa Civil (BR). Lei número N 8.069, de 13 de Julho de 1990 (BR). Dispõe sobre o estatuto da Criança e do adolescente e dá outras providências. http://www.planalto.gov.br/ccivil_03/leis/18069.htm

Carlos, D.M., Campeiz, A.B., Oliveira, W. A., Silva, J.L., Wernet, M., \& Ferriani. M. G. C. (2020). "Não tenho, não tive”: vivências de famílias envolvidas na violência contra crianças e adolescentes. https://www.scielo.br/j/reben/a/k836VpCZRsnQWM6WWN9sKHK/?lang=pt\&format=pdf

Holton, G., Joyner, K., \& Mash, R. (2018). Sexual assault survivors' perspectives on clinical follow-up in the Eden District, South Africa: A qualitative study. https://www-ncbi-nlm-nih.ez182.periodicos.capes.gov.br/pmc/articles/PMC6018593/pdf/PHCFM-10-1631.pdf

Laird, J. J., Klettke, B., Hall, K., Clancy, E., \& Hallford, D. (2020). Demographic and Psychosocial Factors Associated With Child Sexual Exploitation A Systematic Review and Meta-analysis. https://jamanetwork-com.ez182.periodicos.capes.gov.br/journals/jamanetworkopen/fullarticle/2770752

Mitchell, K., Moynihan, M., Pitcher, C., Francis, A., Ingles, A., \& Saewyc, E. (2017). Rethinking research on sexual exploitation of boys: Methodological challenges and recommendations tooptimize future knowledge generation. https://www-sciencedirect.ez182.periodicos.capes.g ov.br/science/article/pii/S0145213417300303?via\%3Dihu

Hailes, P. H., Yu, R., Danese, A., \& Fazel, S. (2019). Long-term outcomes of childhood sexual abuse: an umbrella review. https://www-ncbi-nlmnih.ez182.periodicos.capes.gov.br/pmc/articles/PMC7015702/pdf/EMS85713.pdf

Miranda, M. H. H., Fernandes, F. E. C. V., Melo, R. A., \& Meireles, R. C. (2019). Violência sexual contra crianças e adolescentes: uma análise da prevalência e fatores associados. https://www-ncbi-nlm-nih.ez182.periodicos.capes.gov.br/pmc/articles/PMC6018593/pdf/PHCFM-10-1631.pdf

Moreira. V. S. L. (2020). O papel do enfermeiro no processo de reabilitação psicosocial do sujeito em sofrimento psíquico https://repositorio.unilab.edu.br/jspui/bitstream/123456789/1804/1/2020_arti_vivianmoreira.pdf

Nunes, F. P. (2021). Negligência infantil e seu impacto no desenvolvimento psicossocial. https://repositorio.animaeducacao.c om.br/bitstr eam/ANIMA/17263/1/TC\%20II\%20Paloma\%20-\%20NEGLIG\%c3\%8aNCIA\%20INFANTIL\%20E\%20 SEU\%20IMPACTO\% 20NO\%20DESE NV O LVIMENTO\%20PSICOSSOSSIAL.pdf

Hohendorff, J. V., \& Patias, N. D. (2017). Violência sexual contra crianças e adolescentes: identificação, consequências e indicações de manejo. https://online.unisc.br/seer/index.php/barbaroi/article/view/9474

Pedrosa, A. R. G. (2018). Experiências adversas na infância consequências psicopatológicas e importância de uma boa orientação. https://repositorioaberto.up.pt/bitstream/10216/114011/2/277452.pdf

Pintor, M. A. R. (2017). Abuso sexual infantil: o Educador Social em contexto escolar. https://bibliotecadigital.ipb .pt/bitstream/10198/23144/1/M\%c3\%b3nica\%20Pintor.pdf

Quaglio, W. H., Bueno, S. M. V., Almeida, E. C. (2017). Dificuldades enfrentadas pela equipe de enfermagem no cuidado aos pacientes transplantados: revisão integrativa da literatura. Arq. Ciência Saúde UNIPAR, Umuarama, 21(1), 53-58, jan./abr. 2017.

Sabella, D., (2016). Revisiting Child Sexual Abuse and Survivor Issues. https://pesquisa.bvsalud.org/portal/resource/pt/mdl-26871893

Silva, P. L. N., Veloso, G. S., Queiroz, B. C., Ruas, E. F. G., Alves, C. R., \& Oliveira, V. V. (2021). Desafios da atuação do enfermeiro frente à violência sexual infanto-juvenil. https://docs.bvsalud.org/biblioref/2021/07/1281975/2-desafios-da-atuacao-do-enfermeiro-frente-a-violencia-sexual-_Ml2NTkX.pdf

Soares, A. L. G., Matijasevich, A., Menezes, A. M. B., Assunção, M. C., Wehrmeister, F. C., Howe, L. D., \& Gonçalves, H. (2018). Adverse Childhood Experiences (ACEs) and Adiposity in Adolescents: A Cross-Cohort Comparison. https://onlinelibrary.wiley. com/doi/full/10.1002/oby.22035

Teixeira, S. O. (2019). Atuação da enfermagem frente ao abuso sexual de crianças e adolescentes: Pesquisa Integrativa. https://dspace.uniceplac.edu.br/bitstream/123456789/317/1/Shirniara_Texeira_003424.pdf

Tsuyuki, K., Al-Alusi, N. A., Campbell, J.C., Murry, D., Cimino, A. N., Servin, A. E., \& Stockman, J. K. (2019). Adverse childhood experiences (ACEs) are associated with forced and very early sexual initiation among Black women accessing publicly funded STD clinics in Baltimore, MD. https://www-ncbi-nlmnih.ez182.periodicos.capes.gov.br/pmc/articles/PMC6504039/pdf/pone.0216279.pd 